\title{
Image Segmentation Techniques Using K-Means Clustering to Identify the Land Use Change Detection
}

\author{
Hareesh $B^{1}$, Dr.Vasudeva ${ }^{2}$ \\ 1Assistant Professor, Department of Computer Applications, SJEC, sharsh2000@gmail.com \\ 2Professor, Department of Computer Science, SMVITM, vasudevanitk@gmail.com
}

\begin{abstract}
The change detection of the agriculture land and other land useis one of the important application of remote sensing imagery. The major objective of this paper tomeasure the different boundary regionsof the land classes using an image segmentation techniques. The initial categorizing of different land use classes is experimented by using k-means clustering, which basically clusters the point of interest with the pixel similarity. The measurement of the different pixel region represent the different classes of agriculture area is a challenging task with the real and synthetic images. The important characteristics of the algorithm preserves the cluster pixel details at most of the iterations, however for the similar canopy values the cluster effeminacy varies and the identification of the land clusters also deviates as compared with the ground truth data.
\end{abstract}

Key words: Image Segmentation, Remote Sensing, Graph Based Algorithms, K-Means Clustering

\section{INTRODUCTION}

This century witnessed global revolution in each and every corner of the world. The application of technology in the field of science revolutionized the global economy which indirectly helped and also effected the remote area, especially in India. It is believed that the Indian economy mostly bounded with the agriculture business. But during the last decade more and more science related application are evolved. The remote area is neither deforested nor changed its traditional agriculture crop selection. But in both the way the revolution effected the biological disorders into the environment and now we are witnessing it in the form flooding, draught and seasonal changes. The said advancement also seriously caused the economical disorders in the agriculture sector. Sometime because of very good yields the prices are fallen to the extreme and vice-versa. The fancy pricing for the particular crop initiatesmajority of the farmers to grow the similar crop increases product inflow into market, results in price fall leave the farmers with huge loss. The proposed study focus on to the change detection techniques for the particular crop using remote sensing application.

The rapid advancement in the remote sensing imagery given enormous opportunities to the researchers. The wide scope of application of remote sensing in the field agriculture land cover /land use fulfilled several societal needs in the remote area. The new generation satellite instruments provides images with high spectral and spatial resolution with a global coverage helps in change detection of the agriculture area[1-4].

\section{REMOTE SENSING APPLICATION IN LAND COVER/LAND USE}

The remote sensing application in the field of agriculture is competitively applied from last $10-15$ years to categorize land cover, monitor land-cover, study of vegetation life system and its productivity etc. The increase in number of such applications demands a high accuracy land classifications for the accurate identification of the object of interest. The early advancement in the remote sensing technology started with normalized difference vegetation index (NDVI) for images, but the accuracy characteristics of these images fall short to determine specific vegetation mapping.In the early years of 2000 the MODIS data provided improved spatial and spectral resolution of $250 \mathrm{~m}$ contributed greatly to assess the agriculture change detection. In addition to this the free availability of Landasat data with 30 metre spatial resolution greatly helped to improve the accuracy in agriculture change detection process. In addition to these, the availability of Indian satellite images like IRS, Resourcesat and Awifs series boosted the enormous applications in the field of agriculture and provided an eco-friendly environment for the researchers in the applied sciences[5-11] 


\section{IMAGE SEGMENTATION}

One of the major application of image processing is the segmentation of the images. The remote sensing images were highly used for land use classification. The segmentationof this type of image is used to process and partition a remote sensing image into variable pixel segments. The goal of this segmentation is to streamline some of the land use/land cover issues and/or to identify and publish the change representation of an image from the series of images into something useful to the societal applications. The process is typically used to locate different agriculture species, converted agriculture lands and forest deforestation. The agriculture image segmentation is the process of assigning a label to every similar or dissimilar pixel in an image to identify the similar pixel characteristics, if so it can be grouped with some category of class. In agriculture application a POI compasses with variable pixel intensities, which is to be analysed for the future study.Enormous image segmentation algorithms were used to classify the images, especially with the help ofMatlab tool, the variety of packages will come in handy in image analysis. The Figure 1 shows the different category of image segmentation algorithms[12-14]

\section{PROBLEM STATEMENT}

Most of the time the farmers will start growing or planting same crop with predefined influences caused because of the huge market and sometimes easy maintains of the crop. The study area to assess the change detection selected from certain areas of Dakshina District of Karnataka, India. This coastal area witnessed a huge globalization effects in recent years. The economic standards are improved because of the industrial revolution mainly in the fields of IT sector. The impact of this resulted in the field of agriculture. Most of the time the agriculture areas are converted into building and sometime the said area are changed their crop selection to get more profit. It is sometime because of the unavailability of the agriculture works also. Even though the industrial revolution the advancement in the agriculture is witnessed very low. This effected the agriculture section in huge way. The applied technologies in the field of rice paddy is very limited and the entire process requires a huge manpower, the said areas are converted either into the arecanut plantation or rubber plantation. The nearby high way areas are majorly converted into buildings and industries. Similarly there is a huge revolution in education sector this also increases the building infrastructure and reduces the agriculture and forest areas. The district is also covers large hectares of Western Ghats (WG). The above said revolutions and change in land use developed in a rapid phase caused deforestation in the district[10]. As these changes in the Land use /Land cover visualized and witnessed slowly its effects which can be seen after the decade also. So if there is an automated system to detect such changes to notify the stake holders, the needy actions can be taken to keep the environment healthy and safe. The proposed work

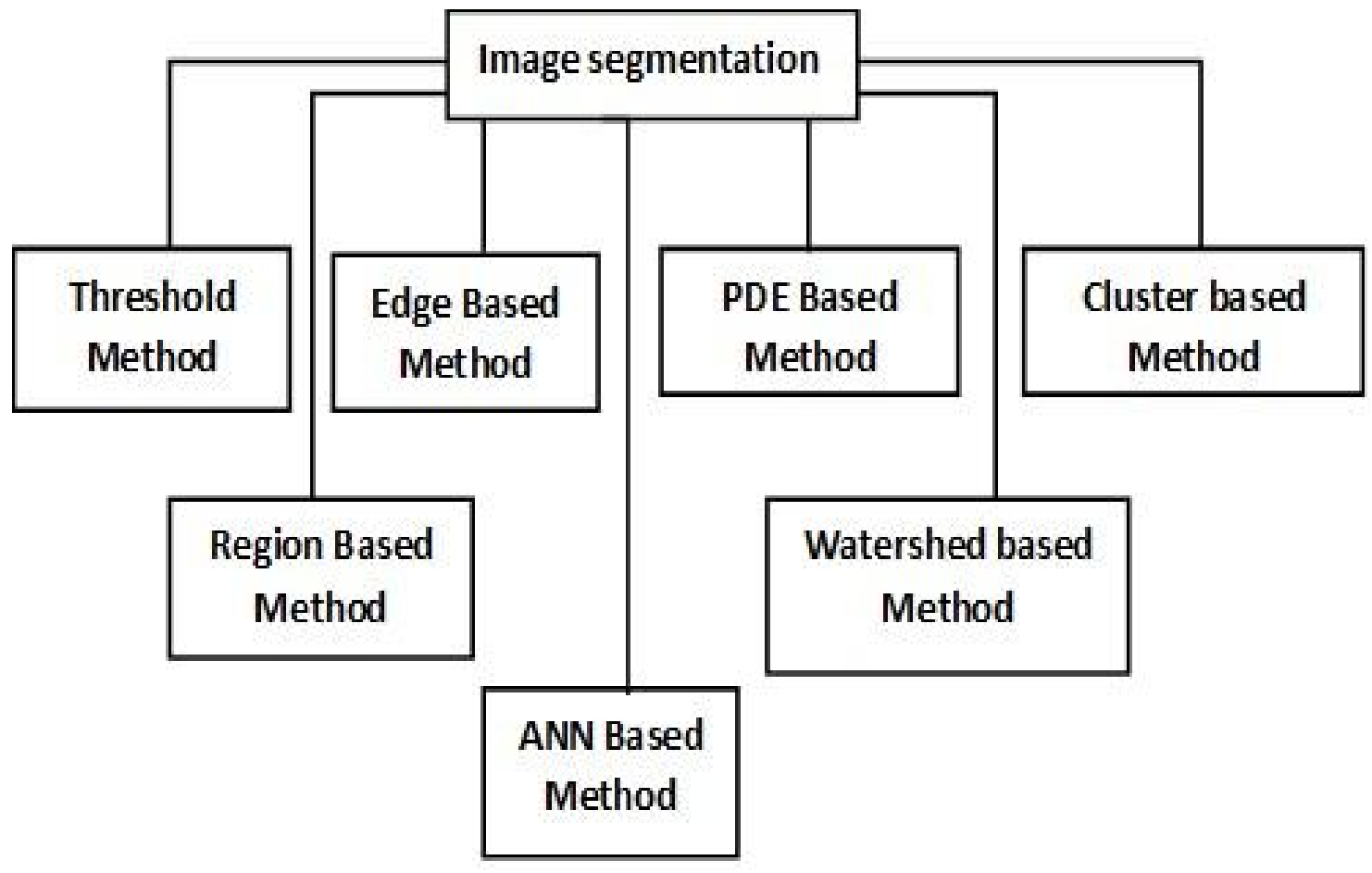

Figure 1: Image Segmentation Types 
carried out to detect such land changes over the years and to come up with an informative system to take decision on the crop selection, timely change in environmental regulations etc. The experimentation in this article made an attempt to quantify different categories of land use for a particular POI taken from the Vamajoor area of ThiruvailGramaPanchyath.

Segmenting a remote sensing image is important for classify different classes of images. The measuring of the different land classes are also important nowadays in different decision making systems. The definition of algorithms for measuring such land classes is important and a cluster of literatures come across comparatively good results by using graph-based image[8].

The objective of the work is to characterize the point of interest (POI) in the sample image. A satellite image of the college campus from the Vamajoor area of ThiruvailGramaPanchyathwas taken and segmentation is performed on the image to identify between the buildings and greenlands and other areas. The final segmented image shows the regions that fall into various clusters and also the image showing the point of interest.

\section{METHODOLOGY}

The solution for the grouping of similar canopy intensities for the agriculture image intensities is a complex process as for some reason the pixel intensity may be similar even if there is a change in agriculture land category. This clustering problem can be solved by using the concept of unsupervised algorithm and its simplest form is $\mathrm{K}$-means clustering[9].

This classification task for the given data set can be accomplished by traversing through a certain clusters with a fixed priori value by defines the $\mathrm{k}$ centroids one for each cluster. The given image dataset is processed by giving a number cluster values initially using the Matlab tool initializes the different centroid values for the different land categories, through this centroid values, it increments with the similar pixel and partitions the cluster when it detects the variant pixel and thus forms the boundary. Thus partitioning helps to classify the required land use and can be measured to identify the change detection over the years[1517].

\section{K-MEANS CLUSTERING}

The segmentation and analysis of remotely sensed data is a key technology to map and monitor the land cover and land use. Over the years these image analysis is experimented with pixel based methods. The recent advancement in tools and technology provides a flexible and efficient methods for object classification. The use of right algorithm along with the high resolution imagery and tools are essential for the monitoring of land cover, such as forestry, agriculture and grasslands. The vector quantization method of K-Means clustering popular in the field of signal processing and data mining and can be generalized to any cluster forming to classify the similarity and dissimilarity criterion. The clustering and partitioning relationship follows the " $n$ " partitioning and " $k$ " clusters with each observation represents the cluster with the nearest mean as a prototype of each cluster. The clusters of different pixel intensities results in some partitioning of some data space[18-23]

The objective function for the clustering hierarchy and partitioning pixel boundary is given in eq 1

$$
J=\sum_{j=1}^{k} \sum_{i=1}^{n}\left\|x_{i}^{(j)}-c_{j}\right\|^{2}
$$

where $\left\|x_{i}^{(j)}-c_{j}\right\|^{\sharp}$ is a chosen distance measure between a data point $\boldsymbol{x}_{i}^{(j)}$ and the cluster centre $s$, is an indicator of the distance of the $\mathrm{n}$ data points from their respective cluster centres.

The algorithm is depicted in the following steps and also shown in Figure 2.

1. The objects of interest represents K points are placed into the initial group centroids.

2. The closest centroid is assigned with an object.

3. Once the steps completes for all the objects, recalculate the position of the $\mathrm{K}$ centroids.

4. Iterate the last two steps until the centroid stops its traversing. This induces the cluster separation as required for the problem statement.

The observations from the analysis results in unsatisfactory classification the clustering may not terminate into the partition even if there is a change in cluster intensity and it is also found that the kmeans algorithm does not necessarily find the most optimal configuration, corresponding to the global objective function minimum. This is because of the sensitivity of the algorithm towards the initial random selection of clusters. The algorithm can be executed multiple times by changing the centroids which once again creates considerable vague in partitions and results in false analysis. It is simple and can be applied to generate a group of minimum clusters. [9]. 


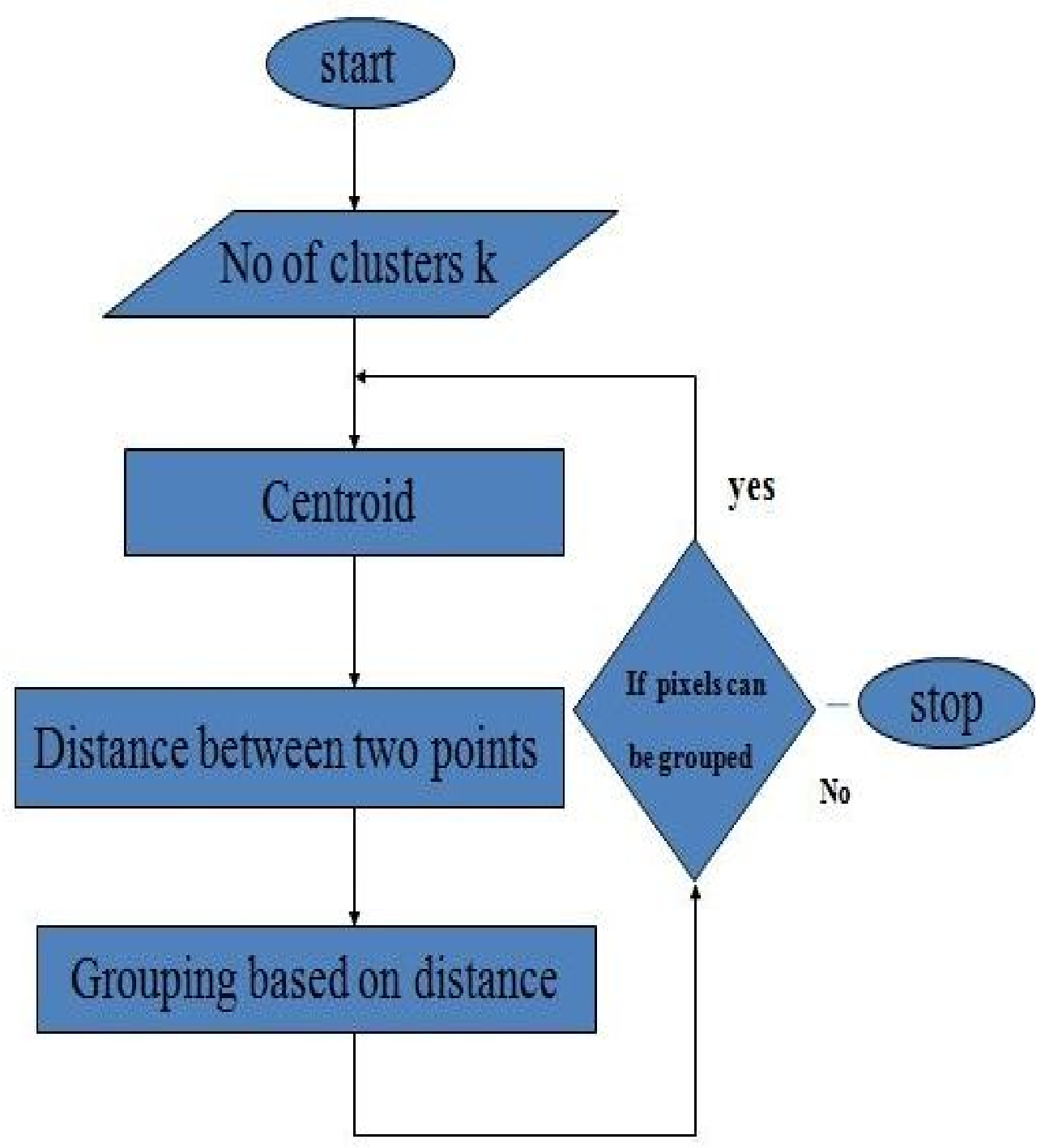

Figure 2: K-Means Algorithm Flowchart

\section{RESULTS AND DISCUSSIONS}

The above algorithm is iteratively applied to the number of images to conclude the image data to be used for the future experimentation as the result gives varied result structure for each set of data as shown in Figure 3. But up to certain extent this algorithm reduces the mixed pixel problem usually generated in this category of pixel based methods. The result is also influenced by the number of samples and the type of samples the pre-processing steps adopted for the image. The 5 clusters unable to characterize the different vegetation blocks, infrastructure development over the period of 2013 to 2018. In this period there was a huge development and consequently the forest and green degradation in the particular POI. The future scope of this work are carried out using graph based image segmentation algorithms and the result analysis enhanced the classification accuracy for input images. 


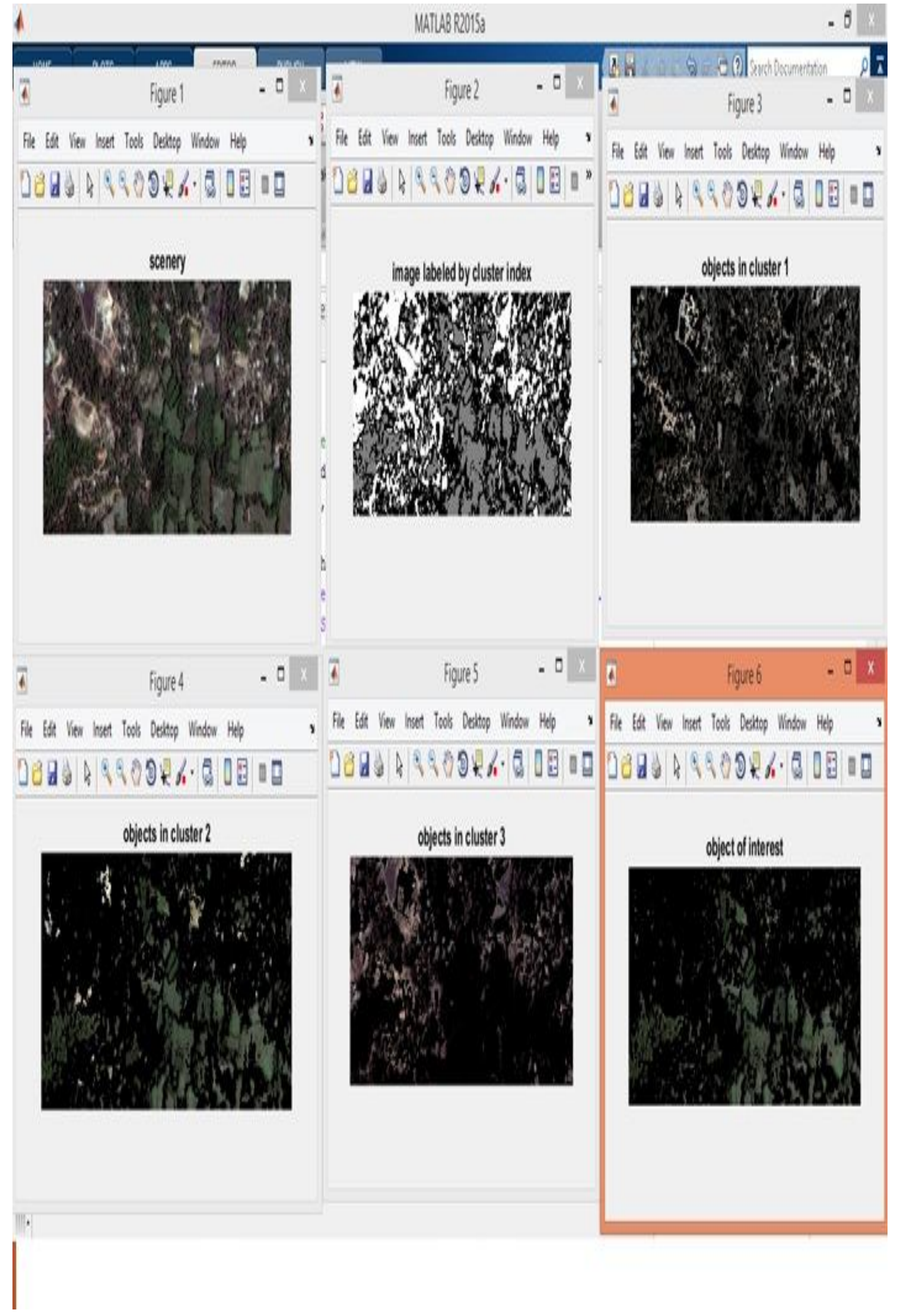

Figure 3: Results of K-Mean Clustering 


\section{CONCLUSION}

Image segmentation remains a challenging problem, however through the sequential steps a substantial progress achieved using k-means algorithms. The resultant segmentation images shown in the Fig. 1 produces deviated results as compared to the ground truth data, because of the problem with the data set. An efficient remote sensing image with the $25 \mathrm{~m}$ resolution may be useful for the efficient classification. Probably various literatures suggests to use LISS IV imaged. Also the accuracy of the results may be compared with other graph based segmentation algorithms like graph-cut etc. which is to be the future work. Image segmentation plays a vital role in digital image processing. The K-means clustering technique is a simple quick algorithm

\section{REFERENCES}

[1] T. W. Ryan, "Image Segmentation Algorithms," Archit. Algorithms Digit. Image Process. II, vol. 0534, p. 172, 1985, doi: $10.1117 / 12.946577$.

[2] J. S. Rawat and M. Kumar, "Monitoring land use/cover change using remote sensing and GIS techniques: A case study of Hawalbagh block, district Almora, Uttarakhand, India," Egypt. J. Remote Sens. Sp. Sci., vol. 18, no. 1, pp. 77-84, 2015, doi: 10.1016/j.ejrs.2015.02.002.

[3] Y. Shao, J. Ren, and J. B. Campbell, Multitemporal remote sensing data analysis for agricultural application, vol. 1-9. Elsevier, 2017.

[4] A. Halder and A. Dasgupta, "Image segmentation using rough set based $\mathrm{K}$ means algorithm," ACM Int. Conf. Proceeding Ser., pp. 53-58, 2012, doi: $10.1145 / 2381716.2381728$.

[5] P. Shanmugapriya, S. Rathika, T. Ramesh, and P. Janaki, "Applications of Remote Sensing in Agriculture - A Review," Int. J. Curr. Microbiol. Appl. Sci., vol. 8, no. 01, pp. 2270-2283, 2019, doi: 10.20546/ijcmas.2019.801.238.

[6] I. S. Sitanggang and H. Harianja, "K-means clustering visualization on agriculture potential data for villages in bogor using mapserver," no. August, pp. 5-6, 2008.

[7] B. Usman and Elixir, "Satellite Imagery Land Cover Classification using K-Means Clustering Algorithm Computer Vision for Environmental Information Extraction," Sci. Engg, vol. 63, no. May, pp. 1867118675, 2013.
[8] M. Arr, "KERALA ENVIRONMENT CONGRESS 2012 Focal Theme at RGCB Auditorium , Thiruvananthapuram Organised by Centre for Environment and Development," no. August 2012, 2015, doi: 10.13140/2.1.3220.1766.

[9] Y. Xie, Z. Sha, and M. Yu, "Remote sensing imagery in vegetation mapping: a review," J. Plant Ecol., vol. 1, no. 1, pp. 923, 2008, doi: 10.1093/jpe/rtm005.

[10] B. Vandana and S. S. Kumar, "Hybrid K Mean Clustering Algorithm for Crop Production Analysis in Agriculture," Int. $J$. Innov. Technol. Explor. Eng., vol. 9, no. 2S, pp. 9-13, 2019, doi: 10.35940/ijitee.b1002.1292s19.

[11] R. P. Sishodia, R. L. Ray, and S. K. Singh, "Applications of remote sensing in precision agriculture: A review," Remote Sens., vol. 12, no. 19, pp. 1-31, 2020, doi: $10.3390 / \mathrm{rs} 12193136$.

[12] M. R. McCall, T. Mehta, C. W. Leathers, and D. M. Foster, "Psyllium husk II: Effect on the metabolism of apolipoprotein B in African green monkeys," Am. J. Clin. Nutr., vol. 56, no. 2, pp. 385-393, 1992, doi: 10.1093/ajen/56.2.385.

[13] P. Roy, S. Goswami, S. Chakraborty, A. T. Azar, and N. Dey, "Image Segmentation Using Rough Set Theory," Int. J. Rough Sets Data Anal., vol. 1, no. 2, pp. 62-74, 2014, doi: 10.4018/ijrsda.2014070105.

[14] J. Shepherd, P. Bunting, and J. Dymond, "Operational Large-Scale Segmentation of Imagery Based on Iterative Elimination," Remote Sens., vol. 11, no. 6, p. 658, 2019, doi: 10.3390/rs11060658.

[15] P. Lemenkova, "Using K-means algorithm classifier for urban landscapes classification in Taipei area, Taiwan Polina Lemenkova To cite this version: HAL Id : hal-02425701," 2019.

[16] S. Patra, S. Ghosh, and A. Ghosh, "Histogram thresholding for unsupervised change detection of remote sensing images," Int. J. Remote Sens., vol. 32, no. 21, pp. 6071-6089, 2011, doi: 10.1080/01431161.2010.507793.

[17] S. SankarNath and P. Rakshit, "A Survey of Image Processing Techniques for Emphysema Detection," Int. J. Comput. Appl., vol. 114, no. 15, pp. 7-13, 2015, doi: 10.5120/20052-1983. 
[18] R. Neware and P. A. Khan, "Identification of agriculture areas in satellite images using Supervised Classification Technique," no. April, 2018.

[19] V. Kumar and S. Agrawal, "Agricultural land use change analysis using remote sensing and GIS: A case study of Allahabad, India," Int. Arch. Photogramm. Remote Sens. Spat. Inf. Sci. - ISPRS Arch., vol. 42, no. 3/W6, pp. 397-402, 2019, doi: 10.5194/isprs-archives-XLII-3-W6-3972019.

[20] F. Guttler, D. Ienco, J. Nin, M. Teisseire, and P. Poncelet, "A graph-based approach to detect spatiotemporal dynamics in satellite image time series," ISPRS J. Photogramm. Remote Sens., vol. 130, pp. 92-107, 2017, doi: 10.1016/j.isprsjprs.2017.05.013.

[21] X. Wang, "Graph based approaches for image segmentation and object tracking To cite this version," Ec. Cent. Lyon, 2015. 